\title{
Chiral phase transition in a random matrix model with three flavors
}

\section{Hirotsugu Fujii}

Institute of Physics, University of Tokyo, Tokyo 153-8902, Japan

\section{Munehisa Ohtani}

Physics Department, School of Medicine, Kyorin University, Tokyo 181-8611, Japan

\section{Takashi Sano*}

Department of Physics, University of Tokyo, Tokyo 113-0033, Japan

Institute of Physics, University of Tokyo, Tokyo 153-8902, Japan

E-mail.tsano@nt1.c.u-tokyo.ac.jp

\begin{abstract}
The chiral phase transition in the conventional random matrix model is the second order in the chiral limit, irrespective of the number of flavors $N_{f}$, because it lacks the $\mathrm{U}_{\mathrm{A}}(1)$-breaking determinant interaction term. Furthermore, it predicts an unphysical value of zero for the topological susceptibility at finite temperatures. We propose a new chiral random matrix model which resolves these difficulties by incorporating the determinant interaction term within the instanton gas picture. The model produces a second-order transition for $N_{f}=2$ and a first-order transition for $N_{f}=3$, and recovers a physical temperature dependence of the topological susceptibility.
\end{abstract}

The XXVII International Symposium on Lattice Field Theory - LAT2009

July 26-31 2009

Peking University, Beijing, China

\footnotetext{
${ }^{*}$ Speaker.
} 


\section{Introduction}

One of the prominent features of non-perturbative QCD is spontaneous breaking of chiral symmetry in the light quark sector. According to the Banks-Casher relation [1], the order parameter $\langle\bar{q} q\rangle$ is related to the Dirac spectral density at zero eigenvalue in the thermodynamic limit. Within the so-called $\varepsilon$ regime characterized by the system size $L^{4}$ such that $m_{\pi} \ll 1 / L \ll 1 \mathrm{GeV}$, the QCD partition function is dominated by the constant pion field configurations. In this regime, universal properties of the Dirac eigenvalue distribution near zero are legitimately analyzed in a chiral random matrix (ChRM) theory [2], where the kinetic term of the Dirac operator is discarded and the complexity of the gauge field dynamics is represented by treating the Dirac operator as a random matrix of constant modes. The matrix size of the constant modes $2 N$ is considered to be proportional to the system volume. By taking a large volume limit $N \rightarrow \infty$, away from the $\varepsilon$ regime, one can study thermodynamics of the ChRM theory as a schematic mean-field model for QCD. One finds the ground state of the model in the chirally broken phase and can explore the model phase diagram by introducing the temperature $t[3]$ and the quark chemical potential $\mu$ [4].

There are two drawbacks, however, in the ChRM model, concerning the $U_{A}(1)$ symmetry. Firstly, the $\mathrm{U}_{\mathrm{A}}(1)$-breaking determinant term is missing in the effective action of the model, which consequently predicts a second-order phase transition at finite temperature for any number of the quark flavors $N_{f}$. Secondly, the topological susceptibility is suppressed unphysically to vanish at finite temperatures.

In an earlier work [5], the appropriate form of the ChRM model with a $\mathrm{U}_{\mathrm{A}}(1)$-breaking term is speculated from a quark model with the determinant interaction in $0+1$ dimensions. In addition to the constant modes in the conventional ChRM model, new constant modes are introduced so as to reproduce the determinant interaction. These new modes are considered to be associated with instantons and called topological zero modes. However the effective potential of the starting quark model is unbounded from below for $N_{f}=3$, and therefore no physical ground state exists.

In this paper we propose a new ChRM model [6] changing the distribution of the topological zero modes, which results in the determinant term appearing under a logarithm. Then the effective potential becomes stable for any $N_{f}$, and describes a second-order phase transition for $N_{f}=2$ while a first-order transition for $N_{f}=3$. Moreover, the topological susceptibility shows physical behavior as a function of temperature in our model.

\section{Chiral random matrix model at finite temperature}

In analogy with the QCD partition function, the ChRM model with $N_{f}$ flavors of mass $m_{f}$ is defined as an average of the quark determinants [3]

$$
Z_{v}=\int d W e^{-N \Sigma^{2} \operatorname{tr} W^{\dagger} W} \prod_{f=1}^{N_{f}} \operatorname{det}\left(D+m_{f}\right),
$$

where the Dirac operator has been replaced with an anti-Hermite matrix of constant modes

$$
D=\left(\begin{array}{cc}
0 & i W+i t \mathbf{1}_{N-|v| / 2} \\
i W^{\dagger}+i t \mathbf{1}_{N-|v| / 2} & 0
\end{array}\right)
$$


with an $(N+v / 2) \times(N-v / 2)$ complex matrix $W$. The Gaussian random distribution of $W$ is a simple realization of the complexity of the gluon dynamics. Chiral symmetry is retained as a fact $\left\{D, \gamma_{5}\right\}=0$ with $\gamma_{5}=\operatorname{diag}\left(1_{N+v / 2},-1_{N-v / 2}\right)$. The temperature effect has been introduced in the Dirac operator with a constant $t$, which may be interpreted as the lowest Matsubara frequency $\pi T$. One can easily show that this matrix $D$ has $|v|$ exact zero eigenvalues, which are interpreted as the zero modes accompanied by the topological charge $v$. The complete partition function is obtained after the sum over $v$ weighted by the quenched topological susceptibility $\tau$ of the pure gluon dynamics:

$$
Z_{\theta}=\sum_{v=-2 N}^{2 N} e^{-\frac{v^{2}}{2(2 N) \tau}} e^{i v \theta} Z_{v}
$$

where the $\theta$ angle has been introduced.

After rewriting the determinant with the fermion variables and doing the Gaussian integral of $W$ in $Z_{v}$, we find a four-fermion vertex interaction, which can be unfolded by introducing an $N_{f} \times N_{f}$ auxiliary variable $S \sim q_{L}^{\dagger} q_{R}$. We perform the fermion integral to obtain an expression for $Z_{v}$ in terms of $S$,

$$
Z_{v}=\int d S e^{-N \Sigma^{2} \operatorname{tr}\left(S S^{\dagger}\right)} \operatorname{det}\left((S+\mathscr{M})\left(S^{\dagger}+\mathscr{M}\right)+t^{2}\right)^{N-\frac{|v|}{2}} \times \begin{cases}\operatorname{det}(S+\mathscr{M})^{v} & (v \geq 0) \\ \operatorname{det}\left(S^{\dagger}+\mathscr{M}^{\dagger}\right)^{-v} & (v<0)\end{cases}
$$

where $\mathscr{M}=\operatorname{diag}\left(m_{1}, \ldots, m_{N_{f}}\right)$.

In the thermodynamic limit we set $v=0$ and evaluate Eq. (2.4) with the saddle point equation. For $S \propto \mathbf{1}_{N_{f}}$ and $\mathscr{M}=0$, the $N_{f}$ dependence is factored out in the saddle point equation, implying a second order phase transition for any number of $N_{f}$. Furthermore, $Z_{v}$ is non-analytic in $v$ as the integrand contains a term with $|v|$, which causes the unphysical suppression of the topological susceptibility at finite temperatures [7]. Note that $|v|$ disappears when $t=0$.

\section{Chiral random matrix model with determinant interaction}

Near-zero modes and topological zero modes - Let us first recall the instanton gas picture. An isolated instanton is a localized object accompanying a right-handed exact fermion zero mode. In a dilute system of $N_{+}$instantons and $N_{-}$anti-instantons, we expect $N_{+}$right-handed and $N_{-}$lefthanded zero modes even at finite temperatures. In an effective theory at long distances, effects of the instantons should be integrated out, which will result in $\mathrm{U}_{\mathrm{A}}(1)$-breaking effective interactions. The fundamental assumption in our model is the classification of the constant modes into the nearzero modes and the topological zero modes [5, 6]. We deal with the $2 N$ near-zero modes appearing in the conventional models and additionally the $N_{+}+N_{-}$topological zero modes which we regard as the modes accompanied by the instantons. In our model, $N_{ \pm}$fluctuate according to the instanton distribution, and the number of the exact zero modes is given by $v=N_{+}-N_{-}$. Eventually we sum over $N_{+}$and $N_{-}$assuming a certain distribution with the mean value of $\mathscr{O}(N)$.

We write down a Gaussian ChRM model for definite numbers of zero modes as [5, 6]

$$
Z_{N_{+}, N_{-}}^{N}=\int d A d B d X d Y e^{-N \Sigma^{2} \operatorname{tr}\left(A A^{\dagger}+B B^{\dagger}+X X^{\dagger}+Y Y^{\dagger}\right)} \prod_{f=1}^{N_{f}} \operatorname{det}\left(D+m_{f}\right)
$$


with

$$
D=\left(\begin{array}{cccc}
0 & i A+i t \mathbf{1}_{N} & 0 & i X \\
i A^{\dagger}+i t \mathbf{1}_{N} & 0 & i Y & 0 \\
0 & i Y^{\dagger} & 0 & i B \\
i X^{\dagger} & 0 & i B^{\dagger} & 0
\end{array}\right)
$$

where the matrix $A$ corresponds to the near-zero modes of the conventional model and the $N_{+} \times N_{-}$ matrix $B$ represents the topological zero modes. The matrices $X$ and $Y$ induce mixing among these modes. Note that the temperature term $t$ is introduced only for the near-zero modes. Following the same steps as in Sec. 2] we find the sigma model representation for this ChRM model, which is analytic in $N_{ \pm}$as well as $N$ unlike Eq. (2.4):

$$
Z_{N_{+}, N_{-}}^{N}=\int d S e^{-N \Sigma^{2} \operatorname{tr} S^{\dagger} S} \operatorname{det}\left[(S+\mathscr{M})\left(S^{\dagger}+\mathscr{M}^{\dagger}\right)+t^{2}\right]^{N} \operatorname{det}(S+\mathscr{M})^{N_{+}} \operatorname{det}\left(S^{\dagger}+\mathscr{M}^{\dagger}\right)^{N_{-}} .
$$

Distribution of the topological zero modes - The complete partition function is obtained after summing $Z_{N_{+}, N_{-}}^{N}$ over the instanton numbers $N_{+}$and $N_{-}$. Here we simply assume independent distributions $P\left(N_{ \pm}\right)$for $N_{+}$and $N_{-}$, i.e.,

$$
Z_{\theta}=\sum_{N_{+}, N_{-}} e^{i v \theta} P\left(N_{+}\right) P\left(N_{-}\right) Z_{N_{+}, N_{-}}^{N}=\int d S e^{-2 N \Omega(S ; t, m, \theta)} .
$$

Let us first consider $P\left(N_{ \pm}\right)$in a dilute instanton gas picture. For a one-instanton configuration, one may assign a weight $\kappa$ compared with a no-instanton configuration, and multiply a factor $N \propto V$ taking into account the integration over the instanton location. For a configuration with $N_{+(-)}$ (anti-)instantons, we then have a Poisson distribution

$$
P_{\mathrm{Po}}\left(N_{ \pm}\right)=\frac{1}{N_{ \pm} !}(\kappa N)^{N_{ \pm}}
$$

where the factorial $N_{+(-)}$! appears as the symmetry factor. The summation with $P_{\mathrm{Po}}\left(N_{ \pm}\right)$in Eq. (3.4) results in the exponentiation of the determinant term [5]:

$$
\Omega_{\mathrm{Po}}=\frac{1}{2} \Sigma^{2} \operatorname{tr} S S^{\dagger}-\frac{1}{2} \ln \operatorname{det}\left[(S+\mathscr{M})\left(S^{\dagger}+\mathscr{M}^{\dagger}\right)+t^{2}\right]-\frac{1}{2} \kappa\left[e^{i \theta} \operatorname{det}(S+\mathscr{M})+e^{-i \theta} \operatorname{det}\left(S^{\dagger}+\mathscr{M}^{\dagger}\right)\right] .
$$

This determinant term is commonly incorporated in effective models as the $\mathrm{U}_{\mathrm{A}}(1)$ anomaly term. However this potential is unbound for $N_{f}=3$ in the ChRM model because the term $\operatorname{det}(S+\mathscr{M}) \sim$ $\phi^{3}$ for large $S=\phi \mathbf{1}_{N_{f}}$ dominates over the other terms in $\Omega_{\mathrm{Po}}$.

It should be noticed here that the fermion coupling distorts the $N_{ \pm}$distribution itself. With including the determinant term of the topological zero modes in Eq. (3.3), the effective distribution for $N_{+}$reads

$$
\widetilde{P}_{\mathrm{Po}}\left(N_{+}\right)=\frac{1}{N_{+} !}(\kappa N d)^{N_{+}}
$$

with $d=|\operatorname{det}(S+\mathscr{M})|$, and similarly for $N_{-}$. This means that the average value of $N_{ \pm}$increases indefinitely with increasing $d \sim \phi^{N_{f}}$ as $\left\langle N_{ \pm}\right\rangle=\kappa N d$. However, the possibility of infinitely many 

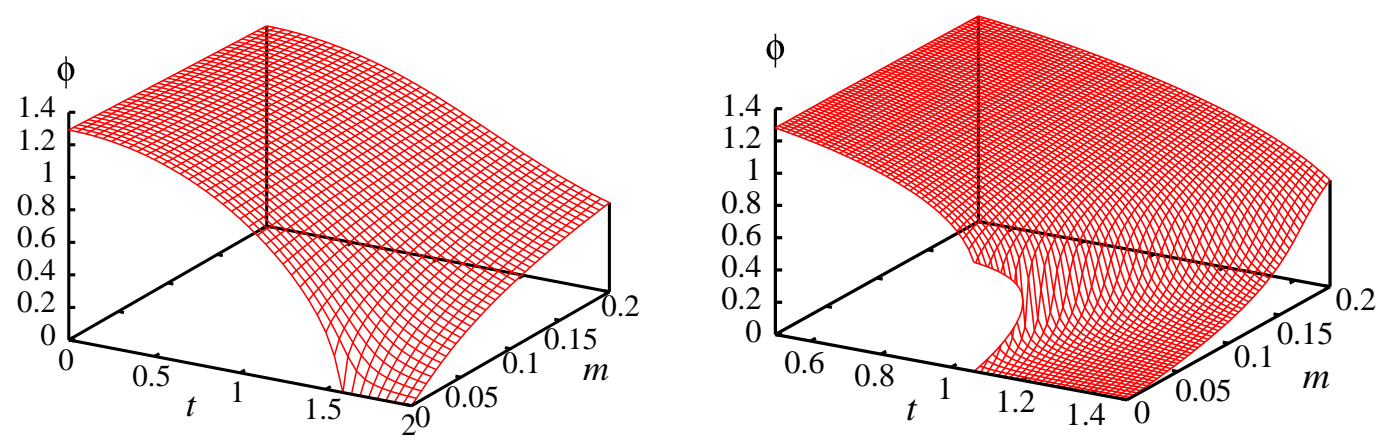

Figure 1: Chiral condensate $\phi$ as a function of $t$ and $m$ for $N_{f}=2$ (left) and 3 (right).

degrees of freedom $N_{ \pm}$within a finite volume is inadequate for a regularized low-energy effective theory. We need a cutoff for $N_{ \pm}$.

Here we set explicitly a maximum value of $\mathscr{O}(N)$ for $N_{ \pm}$. We split the finite space-time volume into $\gamma N$ cells with $\gamma$ being a constant of $\mathscr{O}(1)$, and assign a probability $p$ for a cell to be occupied by a single (anti-)instanton and $(1-p)$ for a cell unoccupied. This assumption results in the binomial distributions for $N_{ \pm}$:

$$
P\left(N_{ \pm}\right)=\left(\begin{array}{c}
\gamma N \\
N_{ \pm}
\end{array}\right) p^{N_{ \pm}}(1-p)^{\gamma N-N_{ \pm}} .
$$

For a small $p$ and a large $\gamma N$, the binomial distribution $P\left(N_{ \pm}\right)$is accurately approximated with the Poisson distribution with the mean $\gamma N p$. But it cannot be a good approximation for a large $p$. The binomial distribution provides a stringent upper bound $\gamma N$ for the number of modes $N_{ \pm}$, in contrast to the Poisson distribution. The corresponding effective potential for $S$ is found to be

$$
\begin{aligned}
\Omega(S ; t, m, \theta)= & \frac{1}{2} \Sigma^{2} \operatorname{tr} S S^{\dagger}-\frac{1}{2} \ln \operatorname{det}\left[(S+\mathscr{M})\left(S^{\dagger}+\mathscr{M}^{\dagger}\right)+t^{2}\right] \\
& -\frac{1}{2} \gamma\left[\ln \left(e^{i \theta} \alpha \operatorname{det}(S+\mathscr{M})+1\right)+\ln \left(e^{-i \theta} \alpha \operatorname{det}\left(S^{\dagger}+\mathscr{M}^{\dagger}\right)+1\right)\right]
\end{aligned}
$$

with $\alpha=p /(1-p)$.

The variance of the topological charge $v=N_{+}-N_{-}$for the binomial distribution is computed as $2 N \tau=2 N \gamma p(1-p)$, where $\tau$ is the quenched topological susceptibility. In the presence of the fermion coupling, this susceptibility will be replaced with

$$
\tilde{\tau}=\gamma \tilde{p}(1-\tilde{p})=\gamma \frac{\alpha d}{(\alpha d+1)^{2}} .
$$

\section{Ground state and fluctuations}

In this section we shall study ground state properties of the system with equal mass, $\mathscr{M}=$ $m \mathbf{1}_{N_{f}}$, for simplicity. Setting $S=\phi \mathbf{1}_{N_{f}}$ with real $\phi$ and with $\theta=0$, we obtain a simple form of the grand potential:

$$
\Omega(\phi ; t, m)=\frac{1}{2} N_{f} \Sigma^{2} \phi^{2}-\frac{1}{2} N_{f} \ln \left[(\phi+m)^{2}+t^{2}\right]-\frac{1}{2} \gamma \ln \left|\alpha(\phi+m)^{N_{f}}+1\right|^{2} .
$$



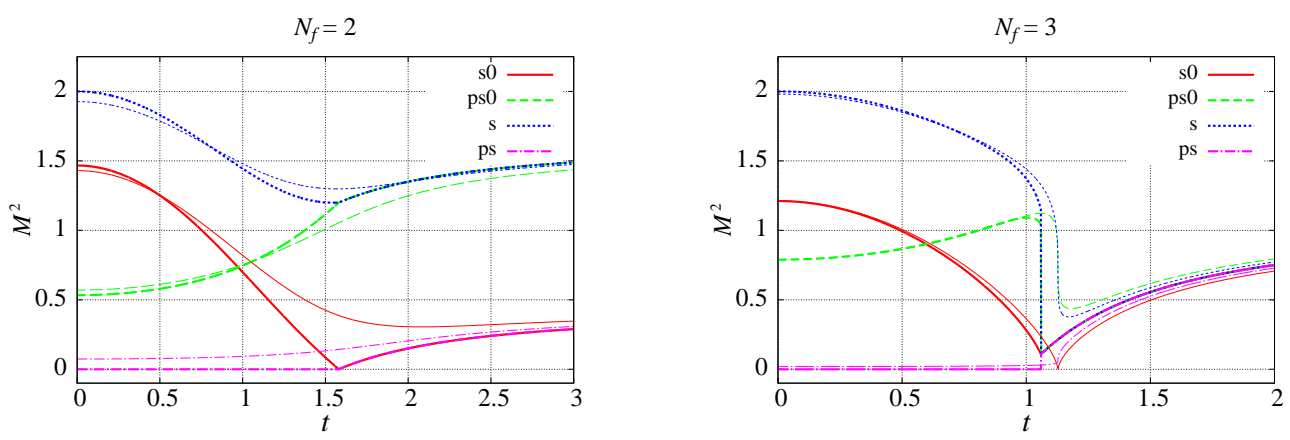

Figure 2: Temperature dependence of the mesonic masses in the flavor-singlet scalar ( $\mathrm{s} 0$ ) and pseudo-scalar (ps0) channels and in the flavor-nonsinglet scalar (s) and pseudo-scalar (ps) channels for $m=0$ (thick lines) and $m \neq 0$ (thin lines). We set $m=0.1\left(m_{c}=0.0265\right)$ as nonzero quark mass for $N_{f}=2$ (3).

The factor $N_{f}$ cannot be factored out in the potential $\Omega$ because of the anomaly term here.

In the thermodynamic limit we calculate the quark condensate $\langle\bar{q} q\rangle \propto \phi$ for $\Sigma=1, \alpha=0.3$ and $\gamma=2$ in Fig. 1. The chiral phase transition is the second order for $N_{f}=2$ in the chiral limit, while it is the first order for $N_{f}=3$ and for $m<m_{c}=0.0265$. The mesonic masses can be defined as $\Omega(S)=\Omega_{0}+\frac{1}{2} M_{\mathrm{s} a}^{2} \sigma^{a 2}+\frac{1}{2} M_{\mathrm{ps} a}^{2} \pi^{a 2}+\cdots$ with $S=\phi+\lambda^{a}\left(\sigma^{a}+i \pi^{a}\right) / \sqrt{2}$ parametrized with $\mathrm{U}(N)$ generators $\left(\operatorname{tr}\left(\lambda^{a} \lambda^{b}\right)=2 \delta^{a b}\right)$. The temperature dependence of the masses are shown in Fig. 2 For pseudo-scalar flavor-nonsinglet masses we find the Gell-Mann-Oakes-Renner relation

$$
(\phi+m)^{2} M_{\mathrm{ps}}^{2}=m \Sigma^{2}(\phi+m) \sim-m\langle\bar{q} q\rangle,
$$

if we identify $\phi+m$ as the pion decay constant $f_{\pi}$. On the other hand, the flavor singlet-masses have an additional contribution from the anomaly term as

$$
M_{\mathrm{s} 0}^{2}=M_{\mathrm{s}}^{2}-\Delta M_{0}^{2}, \quad M_{\mathrm{ps} 0}^{2}=M_{\mathrm{ps}}^{2}+\Delta M_{0}^{2}
$$

with $\Delta M_{0}^{2} \equiv N_{f} \tilde{\tau} /(\phi+m)^{2}$. The would-be Nambu-Goldstone mode becomes massive due to the coupling to the $\mathrm{U}_{\mathrm{A}}(1)$ interaction $\Delta M_{0}^{2}$, and the mass gap is related to the (replaced) quenched susceptibility $\tilde{\tau}$, similarly to the Witten-Veneziano formula.

The topological susceptibility is obtained as $\chi_{\text {top }}=\partial^{2} \Omega(S(\theta) ; \theta) /\left.\partial \theta^{2}\right|_{\theta=0}$ with the saddle point solution $S(\theta)=\phi+i \eta_{0}(\theta) \lambda^{0} / \sqrt{2}$ for small $\theta$, and we find [7]

$$
\frac{1}{\chi_{\text {top }}}=\frac{1}{\tilde{\tau}}+\frac{1}{\tau_{m}} \text {. }
$$

Here $\tilde{\tau}$ is the modified susceptibility defined in Eq. (3.10). The fermion coupling screens $\chi_{\text {top }}$ via the contribution

$$
\tau_{m}=\frac{\Sigma^{2} m(\phi+m)}{N_{f}}=\frac{M_{\mathrm{ps}}^{2}(\phi+m)^{2}}{N_{f}} .
$$

Noting that the $\theta$ dependence can be absorbed into the quark mass term as $m e^{i \theta / N_{f}}$, one can show the axial Ward identity

$$
\chi_{\mathrm{top}}=-\frac{m^{2}}{N_{f}} \chi_{\mathrm{ps} 0}-\frac{m}{N_{f}}\langle\bar{q} q\rangle
$$



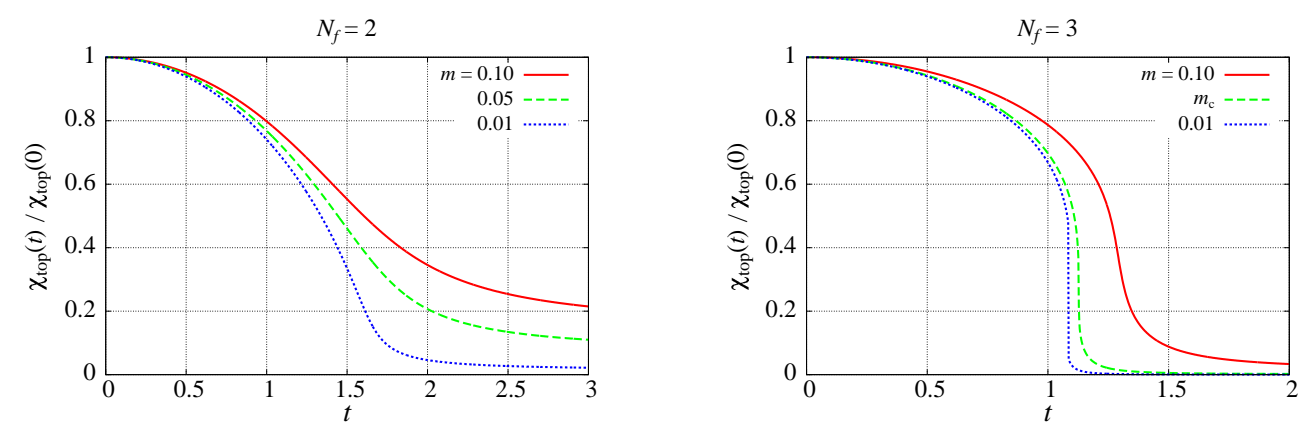

Figure 3: Temperature dependence of topological susceptibility $\chi_{\text {top }}(t)$ for $N_{f}=2$ and 3 .

Since the pseudo-scalar meson in the flavor singlet channel has nonzero mass (4.3) because of the $\mathrm{U}_{\mathrm{A}}$ (1)-breaking term, the pseudo-scalar singlet susceptibility remains finite in the broken phase in the chiral limit. Thus for the small but nonzero quark mass $m$, the decrease of $\chi_{\text {top }}$ follows the chiral condensate $\langle\bar{q} q\rangle \sim \phi$ with increasing $t$, which is clearly observed in Fig. 3.

\section{Summary}

We have presented a chiral random matrix model where the determinant interaction is incorporated by summing up the topological zero modes with the binomial distribution [6]. The model has a stable ground state unlike the model of [5], and describes the $N_{f}$ dependence of the chiral phase transition. At the same time it reproduces the physical temperature dependence of the topological susceptibility. This model can be applied to the $2+1$ flavor case at finite temperature and density [8].

This work is supported in part by Grants-in-Aid of MEXT, Japan (No. 19540269 and 19540273).

\section{References}

[1] T. Banks and A. Casher, Nucl. Phys. B 169 (1980), 103.

[2] E. V. Shuryak and J. J. M. Verbaarschot, Nucl. Phys. A 560 (1993) 306; for review, J. J. M. Verbaarschot and T. Wettig, Ann. Rev. Nucl. Part. Sci. 50 (2000) 343.

[3] A. D. Jackson and J. J. M. Verbaarschot, Phys. Rev. D 53 (1996) 7223; T. Wettig, A. Schäfer and H. A. Weidenmüller, Phys. Lett. B 367 (1996) 28 [Erratum ibid. B 374 (1996) 362].

[4] M. A. Stephanov, Phys. Rev. Lett. 76, 4472 (1996);

A. M. Halasz et al., Phys. Rev. D 58 (1998) 096007.

[5] R.A. Janik, M.A. Nowak and I. Zahed, Phys. Lett. B 392 (1997) 155.

[6] T. Sano, H. Fujii and M. Ohtani, Phys. Rev. D 80 (2009) 034007.

[7] M. Ohtani, C. Lehner, T. Wettig and T. Hatsuda, Mod. Phys. Lett. A 23 (2008) 2465;

C. Lehner, M. Ohtani, J.J.M. Verbaarschot and T. Wettig, Phys. Rev. D 79 (2009) 074016.

[8] H. Fujii and T. Sano, in these proceedings, POS (LAT2009) 189 